(2) Open Access Full Text Article

\title{
Antihyperlipidemic studies of newly synthesized phenolic derivatives: in silico and in vivo approaches
}

This article was published in the following Dove Press journal:

Drug Design, Development and Therapy

\section{Muhammad Tahir Aqeel' \\ Nisar ur-Rahman' \\ Arif-ullah Khan ${ }^{2}$ \\ Zaman Ashraf ${ }^{3}$ \\ Muhammad Latif ${ }^{4}$ \\ Hummera Rafique ${ }^{5}$ \\ Usman Rasheed'}

'Department of Pharmacy, COMSATS Institute of Information Technology Abbottabad, Abbottabad, Pakistan; ${ }^{2}$ Riphah Institute of Pharmaceutical Sciences, Riphah International University, Islamabad, Pakistan; ${ }^{3}$ Department of Chemistry, Allama Iqbal Open University, Islamabad, Pakistan; ${ }^{4}$ College of Medicine, Centre for Genetics and Inherited Diseases (CGID), Taibah University, AI-Madinah Al-Munawwarah, Saudi Arabia;

${ }^{5}$ Department of Chemistry, University of Gujrat, Gujrat, Pakistan
Correspondence: Zaman Ashraf Department of Chemistry, Allama lqbal Open University, $\mathrm{H}-8 / 4$, Islamabad 44000, Pakistan

Tel +9232 I5I9 446I; +92905 7I82

Fax +92 5 |289 |47|

Email mzchem@yahoo.com
Background: Hyperlipidemia is a worth-mentioning risk factor in quickly expanding cardiovascular diseases, including myocardial infarction and, furthermore, in stroke.

Methods: The present work describes the synthesis of phenolic derivatives $4 \mathbf{a}-\mathbf{e}$ and $\mathbf{6 a}-\mathbf{c}$ with the aim of developing antihyperlipidemic agents. The structures of the synthesized compounds were confirmed by spectroscopic data. The in silico docking studies were performed against human 3-hydroxy-3-methylglutaryl-coenzyme A (HMG CoA) reductase enzyme (PDB ID: $1 \mathrm{HWK}$ ), and it was observed that compounds $\mathbf{4 a}$ and $\mathbf{6 a}$ exhibited maximum binding affinity with target protein having binding energies -8.3 and $-7.9 \mathrm{kcal}$, respectively.

Results: Compound 4a interacts with amino acids Val805 with distance $1.89 \AA$ and Met656, Thr558, and Glu559 with bonding distances $2.96,2.70$, and $2.20 \AA$, respectively. The in vivo antihyperlipidemic activity results revealed that compound $\mathbf{4 a}$ indicated minimum weight increment, ie, 20\% compared with 35\% weight increment with standard drug atorvastatin during 6 weeks of treatment. Moreover, increment in high-density lipoprotein cholesterol and decrease in total cholesterol, low-density lipoprotein cholesterol, and triglyceride levels were more prominent in case of $\mathbf{4 a}$ compared to atorvastatin with $P<0.05$. The synthesized compounds were nontoxic and well tolerated because none of the mice were found to suffer from any kind of morbidity and death during 6 weeks of dosing.

Conclusion: Based on our pharmacological evaluation, we may propose that compound 4a may act as a lead structure for the design and development of more potent antihyperlipidemic drugs. Keywords: phenolic derivatives, synthesis, antihyperlipidemic, in silico docking, HMG CoA reductase, atorvastatin

\section{Introduction}

Human beings are persistently confronting life-threatening morbidities such as cardiovascular disorders and cancer. Cardiovascular diseases account for $\sim 17$ million deaths annually worldwide. Above all, atherosclerosis is the major factor for the pathogenesis of myocardial and cerebral infarctions. Increased plasma low-density lipoproteins and triglycerides along with low levels of high-density lipoproteins are often the major root cause of atherosclerosis and ultimately myocardial and cerebral infarctions. Correspondingly, obesity has a direct linkage with atherosclerosis and myocardial infarction. Exact etiology of obesity is relatively unclear, but still the major contributors are genetic, environmental, and dietary factors. ${ }^{1}$

To counter these morbidities, an efficacious and safer pharmacological solution is of most extreme significance. The process of drug discovery is fastidious and expensive. The quest for new effective molecules is challenging yet the accessibility of various $\mathrm{BY}$
NC hereby accept the Terms. Non-commercial uses of the work are permitted without any further permission from Dove Medicat 
docking tools and computer-aided methodologies has limited the gap to a specific level. ${ }^{2,3}$ Antioxidants have phenolic flavonoids that scavenge oxygen radicals and are involved in the inhibition of enzyme activity. Phenolic compounds are auxiliary metabolites present in fruits and vegetables. ${ }^{4,5}$ They possess diverse pharmacological activities including cardiovascular, ${ }^{6}$ antioxidant, ${ }^{5-9}$ antiatherogenic, ${ }^{10,11}$ antiinflammatory, ${ }^{12-16}$ and antithrombotic. ${ }^{17-19}$ Various studies on the experimental animals reported the effect of phenolic compounds such as quercetin and rutinosides on the lipid metabolism causing a decrease in the serum lipid levels. ${ }^{20,21}$

Derivatives of natural poly-phenols have shown remarkable bioactivity in various disease areas both in prevention and treatment. Among numerous classes of polyphenols, hydroxycinnamic acid derivative is the renowned group of phenols found in cereals, roasted coffee, and green vegetables and is reported as a potential antioxidant. ${ }^{22-24}$ Sinapic acid and ferulic acid are the most studied hydroxycinnamic acid derivatives and were found to be highly effective as antioxidant, ${ }^{25,26}$ and in recent data, they are found to be preventive and therapeutic agents in several other diseases including atherosclerosis, inflammatory injury, and cancer. ${ }^{27,28}$ Caffeic acid derivatives are another class of biologically active phenolic derivatives as antioxidant $\mathrm{t}^{29,30}$ and anticancer. ${ }^{31-33}$

Recently, few studies have demonstrated polyphenolic moieties as potential antihyperlipidemic agents by inhibiting 3-hydroxy-3-methylglutaryl-coenzyme A (HMG CoA) reductase in in silico models. ${ }^{34}$ Considering such enormous pharmacological potential, derivatives of natural phenols are exciting prospect for future drug development especially in antihyperlipidemic and cardiovascular therapeutic domains. The use of computational analysis to establish the binding affinity of the drug-like compounds with different enzymes, receptors, and proteins is categorized in in silico pharmacology. ${ }^{35}$ The in silico methods have been frequently used in the discovery and optimization of novel molecules with affinity to a target and in the optimization of pharmacokinetic parameters. ${ }^{36,37}$ Various studies have been reported in which the in silico assessment of the drug-like compounds showed the binding affinity with enzymes, proteins, transporter proteins, and receptors and is very well correlated with their in vitro and in vivo pharmacological effects. ${ }^{38}$ Consequently, the in silico pharmacology paradigm is ongoing and presents a vast platform for the discovery of new drug targets as well as screening of new drug-like compounds that can be considered as drug.

In this perspective, current study is aimed to develop potent antihyperlipidimic agents. The phenolic derivatives $\mathbf{4 a}-\mathbf{e}$ and $\mathbf{6 a}-\mathbf{c}$ have been synthesized and characterized; the in vivo antihyperlipidemic evaluation was carried out.
The in silico docking studies against HMG CoA reductase enzyme (PDB ID: 1HWK) were also performed to compare the dry laboratory results with wet laboratory findings.

\section{Materials and methods Drugs and chemicals}

Atorvastatin was obtained from Global Pharmaceuticals (Islamabad, Pakistan), and mice were received as gift from National Institute of Health (Islamabad, Pakistan).

\section{Synthesis of 2-methoxyphenyl chloroacetate (2)}

The 2-methoxyphenol (1) $(0.01 \mathrm{~mol})$ was dissolved in anhydrous dichloromethane $(25 \mathrm{~mL})$, and triethylamine (0.01 mol) was added slowly. The resulting solution was then cooled in an ice salt mixture to 0 to $-5^{\circ} \mathrm{C}$. The chloroacetyl chloride $(0.01 \mathrm{~mol})$ in dry dichloromethane was added drop wise to the reaction mixture with constant stirring over a period of $1 \mathrm{~h}$ maintaining the temperature constant. The reaction mixture was then stirred at room temperature for further $5 \mathrm{~h}$ and washed with $5 \% \mathrm{HCl}$ and $5 \%$ sodium hydroxide solution. The organic layer was washed with saturated aqueous $\mathrm{NaCl}$, dried over anhydrous magnesium sulfate and filtered, and the solvent was removed under reduced pressure. The crude product was purified by silica gel column to afford the corresponding 2-methoxyphenyl chloroacetate $(2)$ : melting point, $56^{\circ} \mathrm{C}-58^{\circ} \mathrm{C}$; reaction time, $4 \mathrm{~h}$; yield, 78\%; $R_{\mathrm{f}}, 0.62$ (n-hexane:ethyl acetate 3:1); FTIR $v_{\max }(1 / \mathrm{cm}): 2,903(\mathrm{sp} 2 \mathrm{C}-\mathrm{H}), 2,863(\mathrm{sp} 3 \mathrm{C}-\mathrm{H}), 1,719(\mathrm{C}=\mathrm{O}$ ester), 1,589 $(\mathrm{C}=\mathrm{C}$ aromatic $), 1,164(\mathrm{C}-\mathrm{O}$ ester $)$.

\section{General procedure for the synthesis of title compounds $4 a-4 e$ and $6 a-6 c$}

The substituted benzoic acids (3a-3e) $(0.01 \mathrm{~mol})$, triethyl amine $(0.01 \mathrm{~mol})$, and potassium iodide $(0.01 \mathrm{~mol})$ were mixed in dimethylformamide $(25 \mathrm{~mL})$ and stirred at room temperature. The 2-methoxyphenyl chloroacetate intermediate (2) was then added to the reaction mixture slowly and at the same temperature overnight (Scheme 1). The final products $(\mathbf{4 a}-\mathbf{4 e})$ were then extracted with ethyl acetate $(3 \times 25 \mathrm{~mL})$. The combined ethyl acetate layer was then washed with $5 \% \mathrm{HCl}, 5 \%$ sodium carbonate, and finally brine solution. The organic layer was dried over anhydrous magnesium sulfate and filtered, and the solvent was removed under reduced pressure to afford the crude products $(\mathbf{4 a}-\mathbf{4 e})$. The title compounds $(\mathbf{4 a}-\mathbf{4 e})$ were purified by silica gel column chromatography (n-hexane:ethyl acetate $3: 1$ ). The same procedure was used for the preparation of final products $(\mathbf{6 a}-\mathbf{6 c})$ (Scheme 2$)$. 
<smiles>COc1ccccc1O</smiles>

(1)<smiles>COc1ccccc1OC(=O)CCl</smiles>

(2)<smiles>[R]c1cccc(C(=O)NNC(C)(C)COC(=O)c2cc(C(=O)OCC(=O)Oc3ccccc3OC)ccc2[R])c1</smiles>

$\mathrm{R}=$

$\begin{array}{lll}\mathbf{3 a}=3-\mathrm{OH} & \mathbf{3 b}=4-\mathrm{OH} & \mathrm{R} \text { is same as in }(\mathbf{3 a}-\mathbf{3 e}) \\ \mathbf{3 c}=3,4-\mathrm{di}-\mathrm{OH} & \mathbf{3 d}=2,4-\mathrm{di}-\mathrm{OH} & \end{array}$

$3 e=3,5-\mathrm{di}-\mathrm{OH}$

Scheme I Synthesis of phenolic derivatives $(\mathbf{4 a}-\mathbf{4 e})$.

2-(2-Methoxyphenoxy)-2-oxoethyl 3-hydroxybenzoate (4a): solid; melting point, $81^{\circ} \mathrm{C}-83^{\circ} \mathrm{C}$; yield, $76 \% ; R_{\mathrm{f}}, 0.56$ (n-hexane:ethyl acetate 3:1); FTIR $v_{\text {max }}(1 / \mathrm{cm}): 3,248(\mathrm{O}-\mathrm{H})$, 3,965 (sp2 C-H), 2,864 (sp3 C-H), 1,723 (C=O ester), 1,595 $\left(\mathrm{C}=\mathrm{C}\right.$ aromatic), $1,164(\mathrm{C}-\mathrm{O}$ ester $) ;{ }^{1} \mathrm{H} \mathrm{NMR}\left(\mathrm{CDCl}_{3}, \delta \mathrm{ppm}\right)$ : 7.69 (m, 1H, H-6), 7.58 (dd, J=1.6, 1.6 Hz, 1H, H-2), 7.33 (dd, $J=4.8,4.4 \mathrm{~Hz}, 1 \mathrm{H}, \mathrm{H}-5), 7.24$ (m, 1H, H-4), 7.12 (dd, $\left.J=6.4,1.6 \mathrm{~Hz}, 1 \mathrm{H}, \mathrm{H}-6^{\prime}\right), 7.07$ (dd, $J=3.6,1.8 \mathrm{~Hz}, 1 \mathrm{H}, \mathrm{H}-3^{\prime}$ ), 6.95-6.99 (m, 2H, H-4', H-5'), 5.65 (s, 1H, -OH), 5.10 $\left(\mathrm{s}, 2 \mathrm{H},-\mathrm{CH}_{2}\right), 3.85\left(\mathrm{~s}, 3 \mathrm{H},-\mathrm{OCH}_{3}\right) ;{ }^{13} \mathrm{CNMR}\left(\mathrm{CDCl}_{3}, \delta \mathrm{ppm}\right)$; 166.1 ( $\mathrm{C}=\mathrm{O}$ ester), 165.7 ( $\mathrm{C}=\mathrm{O}$ ester), $155.8(\mathrm{C}-3), 150.9$ (C-2'), 139.0 (C-1'), 130.4 (C-5), 129.7 (C-6), 127.3 (C-2), 122.6 (C-1), 122.3 (C-4), 121.4 (C-4'), 120.8 (C-6'), 116.6 (C-3'), $114.5\left(\mathrm{C}-5^{\prime}\right), 61.0\left(-\mathrm{CH}_{2}\right), 55.8\left(-\mathrm{OCH}_{3}\right)$; anal calcd for $\mathrm{C}_{16} \mathrm{H}_{14} \mathrm{O}_{6}$ : C, 63.57; $\mathrm{H}, 4.63$; found $\mathrm{C}, 63.64 ; \mathrm{H}, 4.71$.

2-(2-Methoxyphenoxy)-2-oxoethyl 4-hydroxybenzoate (4b): solid; melting point, $126^{\circ} \mathrm{C}-128^{\circ} \mathrm{C}$; yield, $78 \%$; $R_{\mathrm{f}}$, 0.53 (n-hexane:ethyl acetate 3:1); FTIR $v_{\max }(1 / \mathrm{cm}): 3,149$ (O-H), 2,932 (sp2 C-H), 2,842 (sp3 C-H), 1,718 (C=O ester), 1,593 ( $\mathrm{C}=\mathrm{C}$ aromatic), 1,148 (C-O, ester); ${ }^{1} \mathrm{H}$ NMR
(DMSO- $d_{6}, \delta$ ppm): 7.90 (d, $\left.J=8.8 \mathrm{~Hz}, 2 \mathrm{H}, \mathrm{H}-2, \mathrm{H}-6\right), 7.26$ (ddd, $J=6.8,1.6,1.4 \mathrm{~Hz}, 1 \mathrm{H}, \mathrm{H}-4^{\prime}$ ), 7.11-7.16 (m, 2H, H-3', H-6'), 6.97 (ddd, $J=6.4,1.6,1.21 \mathrm{H}, \mathrm{H}-5^{\prime}$ ), 6.90 (d, $J=8.8 \mathrm{~Hz}$, $2 \mathrm{H}, \mathrm{H}-3, \mathrm{H}-5), 5.15\left(\mathrm{~s}, 2 \mathrm{H},-\mathrm{CH}_{2}\right), 3.78\left(\mathrm{~s}, 3 \mathrm{H},-\mathrm{OCH}_{3}\right), 3.33$ $(\mathrm{s}, 1 \mathrm{H},-\mathrm{OH}) ;{ }^{13} \mathrm{C}$ NMR (DMSO- $\left.d_{6}, \delta \mathrm{ppm}\right): 166.8(\mathrm{C}=\mathrm{O}$ ester), 165.4 ( $\mathrm{C}=\mathrm{O}$ ester), 162.9 (C-4), $151.1\left(\mathrm{C}-2^{\prime}\right), 139.0$ (C-1'), 132.2 (C-2, C-), 127.7 (C-5'), 123.0 (C-1), 121.3 (C-3'), 119.6 (C-4'), 116.0 (C-3, C-5), 113.5 (C-6'), $60.9\left(-\mathrm{CH}_{2}\right), 56.3\left(-\mathrm{OCH}_{3}\right)$; anal calcd for $\mathrm{C}_{16} \mathrm{H}_{14} \mathrm{O}_{6}: \mathrm{C}$, 63.57; H, 4.63; found C, 63.66; H, 4.73.

2-(2-Methoxyphenoxy)-2-oxoethyl 3,4-dihydroxybenzoate (4c): solid; melting point, $154^{\circ} \mathrm{C}-156^{\circ} \mathrm{C}$; yield, $72 \% ; R_{\mathrm{f}}, 0.48$ (n-hexane:ethyl acetate 3:1); FTIR $v_{\text {max }}$ (1/cm): 3,166 (O-H), 2,935 (sp2 C-H), 2,837 (sp3 C-H), 1,722 $(\mathrm{C}=\mathrm{O}$ ester $), 1,600(\mathrm{C}=\mathrm{C}$ aromatic $), 1,148(\mathrm{C}-\mathrm{O}$ ester); ${ }^{1} \mathrm{H}$ NMR (DMSO- $d_{6}, \delta$ ppm): 7.43 (d, $J=2.0 \mathrm{~Hz}$, 1H, H-2), 7.39 (dd, J=6.0, $2.0 \mathrm{~Hz}, 1 \mathrm{H}, \mathrm{H}-6$ ), 7.25 (ddd, $\left.J=3.6,2.0,1.6 \mathrm{~Hz}, 1 \mathrm{H}, \mathrm{H}-4^{\prime}\right), 7.12-7.16$ (m, 2H, H-3', H-6'), 6.97 (ddd, J=4.0, 2.4, $1.6 \mathrm{~Hz}, 1 \mathrm{H}, \mathrm{H}-5^{\prime}$ ), 6.84 (d, $J=8.0 \mathrm{~Hz}, 1 \mathrm{H}, \mathrm{H}-5), 5.13\left(\mathrm{~s}, 2 \mathrm{H},-\mathrm{CH}_{2}\right), 3.80\left(\mathrm{~s}, 3 \mathrm{H},-\mathrm{OCH}_{3}\right)$,

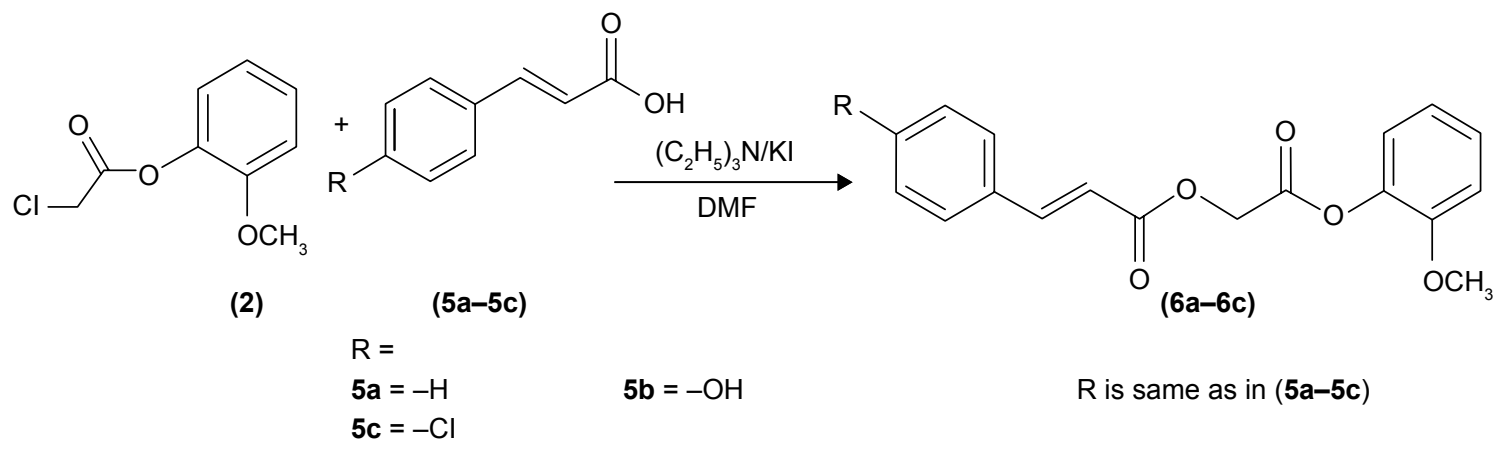

Scheme 2 Synthesis of phenolic derivatives (6a-6c). 
$3.42(\mathrm{~s}, 2 \mathrm{H},-\mathrm{OH}) ;{ }^{13} \mathrm{C}$ NMR (DMSO- $\left.d_{6}, \delta \mathrm{ppm}\right): 171.4(\mathrm{C}=\mathrm{O}$ ester), 166.8 ( $\mathrm{C}=\mathrm{O}$ ester), 165.5 (C-4), 151.4 (C-3), 151.1 (C-2'), $148.1\left(\mathrm{C}-1^{\prime}\right), 139.0\left(\mathrm{C}-5^{\prime}\right), 127.9(\mathrm{C}-6), 123.2\left(\mathrm{C}-4^{\prime}\right)$, 122.7 (C-3'), 121.1 (C-6'), 119.8 (C-2), 116.9 (C-5), 113.5 (C-1), $60.9\left(-\mathrm{CH}_{2}\right), 56.1\left(-\mathrm{OCH}_{3}\right)$; anal calcd for $\mathrm{C}_{16} \mathrm{H}_{14} \mathrm{O}_{7}$ : C, 60.37; H, 4.40; found C, 60.44; H, 4.46.

2-(2-Methoxyphenoxy)-2-oxoethyl 2,4-dihydroxybenzoate (4d): solid; melting point, $109^{\circ} \mathrm{C}-111^{\circ} \mathrm{C}$; yield, $76 \% ; R_{\mathrm{f}}$, 0.46 (n-hexane:ethyl acetate $3: 1)$; FTIR $v_{\max }(1 / \mathrm{cm}): 3,263$ $(\mathrm{O}-\mathrm{H}), 2,942(\mathrm{sp} 2 \mathrm{C}-\mathrm{H}), 2,864(\mathrm{sp} 3 \mathrm{C}-\mathrm{H}), 1,726(\mathrm{C}=\mathrm{O}$ ester), 1,606 ( $\mathrm{C}=\mathrm{C}$ aromatic), 1,162 (C-O ester); ${ }^{1} \mathrm{H}$ NMR (DMSO- $d_{6}, \delta \mathrm{ppm}$ ): 7.73 (d, $\left.J=8.8 \mathrm{~Hz}, 1 \mathrm{H}, \mathrm{H}-6\right), 7.24-7.29$ (m, 2H, H-3', H-6'), 7.15 (ddd, J=6.4, 5.6, 1.6, Hz, 1H, $\mathrm{H}-4^{\prime}$ ), 6.98 (ddd, $J=5.2,3.6,2.0, \mathrm{~Hz}, 1 \mathrm{H}, \mathrm{H}-5^{\prime}$ ), 6.43 (dd, $J=6.4,2.4 \mathrm{~Hz}, 1 \mathrm{H}, \mathrm{H}-3), 6.34$ (d, $J=2.0 \mathrm{~Hz}, 1 \mathrm{H}, \mathrm{H}-5), 5.20$ (s, $\left.2 \mathrm{H},-\mathrm{CH}_{2}\right), 3.78\left(\mathrm{~s}, 3 \mathrm{H},-\mathrm{OCH}_{3}\right), 3.44(\mathrm{~s}, 2 \mathrm{H},-\mathrm{OH}) ;{ }^{13} \mathrm{C}$ NMR (DMSO- $d_{6}, \delta$ ppm): $168.3(\mathrm{C}=\mathrm{O}$ ester), $166.5(\mathrm{C}=\mathrm{O}$ ester), 165.1 (C-2), 163.2 (C-4), 151.1 (C-2'), 139.0 (C-1'), 132.5 (C-5'), 127.8 (C-6), 123.2 (C-4'), 121.3 (C-3'), 119.6 (C-6'), 116.0 (C-3), 109.0 (C-5), 103.1 (C-1), $61.1\left(-\mathrm{CH}_{2}\right)$, $56.1\left(-\mathrm{OCH}_{3}\right)$; anal calcd for $\mathrm{C}_{16} \mathrm{H}_{14} \mathrm{O}_{7}: \mathrm{C}, 60.37 ; \mathrm{H}, 4.40$; found $\mathrm{C}, 60.41 ; \mathrm{H}, 4.48$.

2-(2-Methoxyphenoxy)-2-oxoethyl 3,5-dihydroxybenzoate (4e): solid; melting point, $173^{\circ} \mathrm{C}-175^{\circ} \mathrm{C}$; yield, $84 \% ; R_{\mathrm{f}}$, 0.45 (n-hexane:ethyl acetate 3:1); FTIR $v_{\text {max }}(1 / \mathrm{cm}): 3,134$ $(\mathrm{O}-\mathrm{H}), 2,919$ (sp2 C-H), 2,838 (sp3 C-H), 1,729 (C=O ester), 1,608 ( $\mathrm{C}=\mathrm{C}$ aliphatic), 1,598 ( $\mathrm{C}=\mathrm{C}$ aromatic), 1,145 $\left(\mathrm{C}-\mathrm{O}\right.$, ester); ${ }^{1} \mathrm{H}$ NMR (DMSO- $d_{6}, \delta$ ppm): 7.27 (ddd, $J=5.6$, 3.6, 1.6 Hz, 1H, H-4'), 7.12-7.15 (m, 2H, H-3', H-6'), 6.96 (ddd, $J=6.4,6.4,2.4 \mathrm{~Hz}, 1 \mathrm{H}, \mathrm{H}-5^{\prime}$ ), 6.89 (d, $J=2.4 \mathrm{~Hz}$, 2H, H-2, H-6), 6.48 (dd, J=2.4, $1.6 \mathrm{~Hz}, 1 \mathrm{H}, \mathrm{H}-4), 5.17$ $\left(\mathrm{s}, 2 \mathrm{H},-\mathrm{CH}_{2}\right), 3.78\left(\mathrm{~s}, 3 \mathrm{H},-\mathrm{OCH}_{3}\right), 3.33(\mathrm{~s}, 2 \mathrm{H},-\mathrm{OH})$; ${ }^{13} \mathrm{C}$ NMR (DMSO- $d_{6}, \delta$ ppm); $166.6(\mathrm{C}=\mathrm{O}$ ester $), 165.6$ (C=O ester), 159.1 (C-3, C-5), $151.1\left(\mathrm{C}-2^{\prime}\right), 139.0\left(\mathrm{C}-1^{\prime}\right)$, 130.7 (C-5'), 127.8 (C-2, C-6), 123.0 (C-4'), 121.1 (C-3'), 113.5 (C-6'), 108.2 (C-4), 107.8 (C-1), $61.3\left(-\mathrm{CH}_{2}\right), 56.3$ (C-1"); anal calcd for $\mathrm{C}_{16} \mathrm{H}_{14} \mathrm{O}_{7}: \mathrm{C}, 60.37 ; \mathrm{H}, 4.40$; found $\mathrm{C}$, $60.43 ; \mathrm{H}, 4.47$.

2-(2-Methoxyphenoxy)-2-oxoethyl (2E)-3-phenylprop-2enoate (6a): solid; melting point, $76^{\circ} \mathrm{C}-78^{\circ} \mathrm{C}$; yield, $85 \% ; R_{\mathrm{f}}$, 0.56 (n-hexane:ethyl acetate $3: 1)$; FTIR $v_{\text {max }}(1 / \mathrm{cm}): 2,938$ (sp2 C-H), 2,835 (sp3 C-H), 1,719 (C=O ester), 1,599 (C=C aromatic), 1,164 (C-O, ester); ${ }^{1} \mathrm{H}$ NMR $\left(\mathrm{CDCl}_{3}, \delta \mathrm{ppm}\right)$ : 7.85 (d, $J=16.0 \mathrm{~Hz}, 1 \mathrm{H}, \mathrm{H}-2), 7.58$ (dd, $J=4.4,1.6 \mathrm{~Hz}$, 2H, H-2', 6'), 7.41-7.43 (m, 3H, H-3', H-4', H-5'), 7.24 (dd, $\left.J=6.0,2.4 \mathrm{~Hz}, 1 \mathrm{H}, \mathrm{H}-3^{\prime \prime}\right), 7.13$ (dd, $J=6.4,2.4 \mathrm{~Hz}, 1 \mathrm{H}$, H-4"), 6.96-6.99 (m, 2H, H-4", H-5"), 6.56 (d, J=16.0 Hz, $1 \mathrm{H}, \mathrm{H}-1), 5.04$ (s, $\left.2 \mathrm{H},-\mathrm{CH}_{2}\right), 3.86\left(\mathrm{~s}, 3 \mathrm{H},-\mathrm{OCH}_{3}\right) ;{ }^{13} \mathrm{C} \mathrm{NMR}$
$\left(\mathrm{CDCl}_{3}, \delta \mathrm{ppm}\right) ; 167.7$ (C=O ester), $166.1(\mathrm{C}=\mathrm{O}$ ester $), 150.9$ (C-2"), 146.3 (C-2), 139.2 (C-1"), 134.1 (C-5"), 130.0 (C-2', C-6'), 128.9 (C-3', C-5'), 128.2 (C-4'), 127.2 (C-1'), 122.6 $\left(\mathrm{C}-3^{\prime \prime}\right), 120.7$ (C-4"), 116.7 (C-6"), $112.5(\mathrm{C}-1), 60.5\left(-\mathrm{CH}_{2}\right)$, $55.9\left(-\mathrm{OCH}_{3}\right)$; anal calcd for $\mathrm{C}_{18} \mathrm{H}_{16} \mathrm{O}_{5}: \mathrm{C}, 69.23 ; \mathrm{H}, 5.12$; found $\mathrm{C}, 69.29 ; \mathrm{H}, 5.17$.

2-(2-Methoxyphenoxy)-2-oxoethyl (2E)-3-(4hydroxyphenyl)prop-2-enoate (6b): solid; melting point, $101^{\circ} \mathrm{C}-103^{\circ} \mathrm{C}$; yield, $78 \% ; R_{\mathrm{f}}, 0.47$ (n-hexane:ethyl acetate 3:1); FTIR $v_{\text {max }}(1 / \mathrm{cm}): 3,132(-\mathrm{OH}), 2,931(\mathrm{sp} 2 \mathrm{C}-\mathrm{H}), 2,849$ $(\mathrm{sp} 3 \mathrm{C}-\mathrm{H}), 1,728(\mathrm{C}=\mathrm{O}), 1,609(\mathrm{C}=\mathrm{C}$ aromatic $), 1,148(\mathrm{C}-\mathrm{O}$ ester); ${ }^{1} \mathrm{H}$ NMR (DMSO- $d_{6}, \delta$ ppm): $7.70(\mathrm{~d}, J=16.0 \mathrm{~Hz}$, $1 \mathrm{H}, \mathrm{H}-2), 7.62$ (d, J=8.8 Hz, 2H, H-2', 6'), 7.26 (ddd, $\left.J=7.6,6.0,1.6 \mathrm{~Hz}, 1 \mathrm{H}, \mathrm{H}-4^{\prime \prime}\right), 7.11-7.16$ (m, 2H, H-3", H-6"), 6.98 (ddd, J=7.6, 6.0, $\left.1.6 \mathrm{~Hz}, 1 \mathrm{H}, \mathrm{H}-5^{\prime \prime}\right), 6.80$ (d, $\left.J=8.8 \mathrm{~Hz}, 2 \mathrm{H}, \mathrm{H}-3^{\prime}, \mathrm{H}-5^{\prime}\right), 6.54(\mathrm{~d}, J=16.0 \mathrm{~Hz}, 1 \mathrm{H}, \mathrm{H}-1), 5.06$ (s, $\left.2 \mathrm{H},-\mathrm{CH}_{2}\right), 3.78\left(\mathrm{~s}, 3 \mathrm{H},-\mathrm{OCH}_{3}\right), 3.33(\mathrm{~s}, 1 \mathrm{H},-\mathrm{OH}) ;{ }^{13} \mathrm{C}$ NMR (DMSO- $d_{6}, \delta$ ppm): $166.1(\mathrm{C}=\mathrm{O}$ ester), $165.8(\mathrm{C}=\mathrm{O}$ ester), 150.9 (C-2"), 144.8 (C-2), 139.1 (C-1"), 132.6 (C-4'), 129.5 (C-2', C-6'), 129.3 (C-3', C-5'), 127.2 (C-5"), 122.7 $\left(\mathrm{C}-1^{\prime}\right), 117.4\left(\mathrm{C}-3^{\prime \prime}\right), 112.6\left(\mathrm{C}-4^{\prime \prime}\right), 112.5\left(\mathrm{C}-6^{\prime \prime}\right), 60.6\left(-\mathrm{CH}_{2}\right)$, $55.9\left(-\mathrm{OCH}_{3}\right)$; anal calcd for $\mathrm{C}_{18} \mathrm{H}_{16} \mathrm{O}_{6}: \mathrm{C}, 65.85 ; \mathrm{H}, 4.87$; found $\mathrm{C}, 65.93 ; \mathrm{H}, 4.93$.

2-(2-Methoxyphenoxy)-2-oxoethyl (2E)-3-(4-chlorophenyl)prop-2-enoate $(\mathbf{6 c})$ : solid; melting point, $73^{\circ} \mathrm{C}-75^{\circ} \mathrm{C}$; yield, $83 \% ; R_{\mathrm{f}}, 0.55$ (n-hexane:ethyl acetate $3: 1$ ); FTIR $v_{\max }(1 / \mathrm{cm}): 2,919(\mathrm{sp} 2 \mathrm{C}-\mathrm{H}), 2,862(\mathrm{sp} 3 \mathrm{C}-\mathrm{H}), 1,725$ $(\mathrm{C}=\mathrm{O}$ ester $), 1,611(\mathrm{C}=\mathrm{C}$ aromatic $), 1,156(\mathrm{C}-\mathrm{O}$ ester $)$; ${ }^{1} \mathrm{H}$ NMR $\left(\mathrm{CDCl}_{3}, \delta \mathrm{ppm}\right): 7.79$ (d, $\left.J=16.0 \mathrm{~Hz}, 1 \mathrm{H}, \mathrm{H}-2\right)$, 7.50 (d, $\left.J=7.6 \mathrm{~Hz}, 2 \mathrm{H}, \mathrm{H}-2^{\prime}, 6^{\prime}\right), 7.43$ (d, J=7.6 Hz, 2H, $\left.\mathrm{H}-3^{\prime}, \mathrm{H}-5^{\prime}\right), 7.24$ (dd, J=6.0, $\left.1.6 \mathrm{~Hz}, 1 \mathrm{H}, \mathrm{H}-3^{\prime \prime}\right), 7.11$ (dd, J=6.4, 1.6 Hz, 1H, H-6"), 6.95-6.99 (m, 2H, H-4", $\left.\mathrm{H}-5^{\prime \prime}\right), 6.57$ (d, J=16.0 Hz, 1H, H-1), 5.06 (s, 2H, $-\mathrm{CH}_{2}$ ), $3.86\left(\mathrm{~s}, 3 \mathrm{H},-\mathrm{OCH}_{3}\right) ;{ }^{13} \mathrm{C} \mathrm{NMR}\left(\mathrm{CDCl}_{3}, \delta \mathrm{ppm}\right) ; 166.1$ $(\mathrm{C}=\mathrm{O}$ ester $), 165.8(\mathrm{C}=\mathrm{O}$ ester$), 151.0\left(\mathrm{C}-2^{\prime \prime}\right), 144.9(\mathrm{C}-2)$, 139.1 (C-1"), 136.5 (C-4'), 132.6 (C-2', C-6'), 129.5 (C-3', C-5'), 127.3 (C-5"), 122.7 (C-1'), 121.6 (C-3"), 120.7 (C-4"), $117.3\left(\mathrm{C}-6^{\prime \prime}\right), 60.6\left(-\mathrm{CH}_{2}\right), 56.0\left(-\mathrm{OCH}_{3}\right)$; anal calcd for $\mathrm{C}_{18} \mathrm{H}_{15} \mathrm{O}_{5} \mathrm{Cl}$ : C, 62.33; $\mathrm{H}, 4.32$; found $\mathrm{C}, 62.41 ; \mathrm{H}, 4.39$.

\section{Antihyperlipidemic studies}

An aggregate of 66 mice (Balb C, male, 25-30 g, aged 4-6 weeks) was obtained, kept in National Institute of Health, and left to be acclimatized for 1 week before starting the investigation. Animal experiments were performed in accordance with the Institute of Laboratory Animal Resources, Commission on Life Sciences, National Research Council (1996), approved by Ethical Committee 
of Riphah Institute of Pharmaceutical Sciences (ref no REC/RIPS/2017/001). Mice were divided into 11 groups, each having six mice. Mice were kept in normal laboratory conditions of temperature $23^{\circ} \mathrm{C} \pm 1{ }^{\circ} \mathrm{C}$ and ambient humidity $55 \% \pm 5 \%$. Mice were housed in stainless steel cages individually and kept in an isolated room. Lights were maintained on an artificial $12 \mathrm{~h}$ light-dark cycle. All mice were weighed weekly during 6 weeks of experimental period. Animal care was in accordance with the guidelines established and approved by the National Institute of Health. Mice were fed with high-fat diet (HFD) mentioned in Table 1 containing cholesterol, cholic acid, peanut oil, and normal laboratory diet for 6 weeks. Standard drug (atorvastatin in this study) and test compounds from $4 \mathbf{a}-\mathbf{e}$ and $\mathbf{6 a}-\mathbf{c}$ were mixed with HFD, and the composition of the test compounds was $10 \mathrm{mg} / \mathrm{kg}$. Experimental compounds from $\mathbf{4 a - e}$ and $\mathbf{6 a}-\mathbf{c}$ were mixed with HFD, and the dose of the test compounds was $10 \mathrm{mg} / \mathrm{kg}$. Group I received normal laboratory diet, Group II received HFD, Group III received standard drug (atorvastatin) along with HFD, and Groups IV-XI received test compounds from 4a-e and $\mathbf{6 a}-\mathbf{c}$, respectively, along with HFD. Mice had free access to feed and water throughout the study. The specified quantity of test compounds and standard was dissolved in $1 \mathrm{~mL}$ of DMSO and mixed in pellet diet. All mice were weighed weekly during 6 weeks of experimental period. The activity was continued for 6 weeks under controlled laboratory conditions (temperature $23^{\circ} \mathrm{C} \pm 1{ }^{\circ} \mathrm{C}$ and ambient humidity $55 \% \pm 5 \%) .{ }^{39}$

\section{Blood collection and analysis}

At the end of the experiment, the animals were anesthetized. Blood samples were collected from the retro orbital vein. The serum was separated by centrifugation at $2,500 \times g$ for $15 \mathrm{~min}$ at $4^{\circ} \mathrm{C}$. The amount of serum triglyceride, total cholesterol, highdensity lipoprotein (HDL) cholesterol, and low-density lipoprotein (LDL) cholesterol were assayed automatically using an ADVIA 1650 lipid analyzer (Bayer, Wuppertal, Germany).

Table I Fat diet composition

\begin{tabular}{ll}
\hline Ingredients & Percentage \\
\hline Cholesterol & 2 \\
Cholic acid & 1 \\
Peanut oil & 5 \\
Wheat flour & 27 \\
Wheat baran & 27 \\
Dry skimmed milk & 20 \\
Fish meal & 15 \\
Common salt & 0.5 \\
Molasses & 2 \\
Mineral + vitamin mix & 0.5 \\
\hline
\end{tabular}

\section{Histopathological evaluation}

Animals were euthanized under ether anesthesia, and the liver was dissected out immediately and weighed. For histopathological analysis, the liver and adipose tissues were fixed in $10 \%$ formalin at room temperature. Adipose tissues used were collected from subcutaneous fat of the abdomen of mice. The tissue was embedded in paraffin, sectioned into 3-4 $\mu \mathrm{m}$ thickness and mounted on the glass microscope slides using standard histological techniques. The sections were stained with hematoxylin-eosin and examined using light microscopy at 200× magnitudes. These light microscopic fields were assessed by an image analyzer on each section. ${ }^{40}$

\section{Docking studies}

\section{Acquisition and preparation of target protein}

Crystal structure of HMG CoA reductase was acquired from Protein Data Bank as PDB format (PDB ID: 1HWKHomosapien) (www.rcsb.org/pdb). ${ }^{41}$ Crystal structure of target protein was validated using the MolProbity server. ${ }^{42}$ Ligands and water molecules were removed from crystal structure using the Accelrys Discovery Studio, and the energy of protein was minimized using the UCSF Chimera. ${ }^{43}$ Active binding site studies were viewed using the PyMOL (Python molecular visualization) software. ${ }^{44}$

\section{Preparation of ligand}

Structures of phenolic experimental compounds were drawn in MarvinSketch tools and were cleaned into 3D using Marvin Space and were downloaded in the PDB file extension. ${ }^{45}$

\section{Docking run}

Docking is a virtual screening tool used to predict in silico interaction of ligand with enzyme and its possible inhibition. Both ligand and target protein were converted from PDB to PDBQT files after the addition of hydrogen and removal of water molecules. Energies of ligand and enzyme were minimized using the UCSF Chimera. These prepared molecules were uploaded as ligand, macromolecules in PyRx, a Pythonbased AutoDock Vina-based scoring function screening tool, autogrid dimension were calculated for $\mathrm{HMG}$ CoA reductase ( $X=147.451, Y=87.546$, and $Z=3.341)$, and inhibitor and target were geometrically optimized and docked. ${ }^{46,47}$

\section{Statistical analysis}

For the determination of change in body weight, liver weight, plasma lipids, and HDL and LDL-cholesterol levels, individual mouse was considered as an experimental unit. All data were analyzed by IBM SPSS 20 package for one-way 


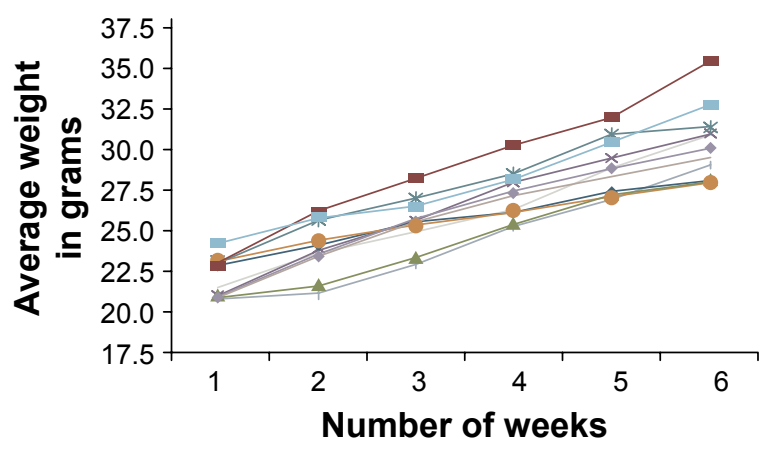

\begin{tabular}{|c|c|c|c|}
\hline$\because N D$ & $\rightarrow-H F D$ & $-\leftarrow$ Std & $\leftarrow 4 \mathrm{c}$ \\
\hline$\rightarrow 4 \mathrm{e}$ & $-4 a$ & $-1-\mathbf{4 b}$ & $-6 a$ \\
\hline$-4 d$ & $\multimap 6 c$ & $--6 \mathbf{b}$ & \\
\hline
\end{tabular}

Figure I Impact of phenolic derivatives on body weight changes on mice supplemented with high-fat diet.

Note: Mean weight in each group is given ( $\mathrm{n}=6$ for each group).

Abbreviations: ND, normal diet; HFD, high-fat diet; Std, reference standard (atorvastatin).

analysis of variance (ANOVA) testing; post hoc Tukey test was used with $P$-value $<0.05$ considering as statistically significant. ${ }^{48}$

\section{Results and discussion Body weights}

Among dietary factors, an increase in carbohydrate and fats in diet has been associated with obesity. ${ }^{49}$ Similarly, few researchers have reported that the differences among strains with the ability of HFD to promote obesity have been demonstrated in both rats and mice. ${ }^{32}$ Some strains of mice such as $\mathrm{A} / \mathrm{J}$ and C57BL do develop obesity when fed on HFD. Meanwhile, SWR mice were resistant to develop obesity when fed on similar HFD. In the present study, mice fed with a HFD demonstrated predictable weight, cholesterol, triglycerides, and LDL increment with a decrease in HDL levels after 6 weeks in the hyperlipidemic control group (HFD group) (Figure 1). After 6 weeks, mice fed with 4a experimental compound indicated least weight increase, ie, $20 \%$ compared with $35 \%$ weight increase with atorvastatin. Even 4e, 6a, and $\mathbf{6 b}$ showed comparable weight increment vs atorvastatin, ie, 36, and 35\%, respectively (Figure 2). These results propose that weight increment in case of $4 \mathbf{a}$ was significantly $(P<0.05)$ less than reference standard and increase in mice weight in case of $4 \mathbf{e}, \mathbf{6 a}$, and $\mathbf{6 b}$ was insignificant but comparable with reference standard (Figure 2).

\section{Plasma lipid profile}

As already discussed, an increase in serum cholesterol and LDL levels may lead to atherosclerosis and ultimately to myocardial infarction. Raised serum triglycerides (TGs) have also been reported to have a role in the aggravation of atherosclerosis..$^{50}$ Meanwhile various studies have shown that drugs decreasing serum TG, LDL, and cholesterol levels reduce the risk of coronary artery diseases. ${ }^{51}$ Various phenols including gallic acid and linoleic acid have been found to be antiatherogenic especially in in vivo green tea extracts reducing serum TGs and cholesterol as well as stimulating energy expenditure, fat oxidation, and fecal lipid oxidation..$^{52}$

In this study, it was observed that all experimental molecules were able to decrease cholesterol, LDL, and triglycerides and increase HDL in hyperlipidemic mice, but in particular, 4a showed highly significant reduction in serum

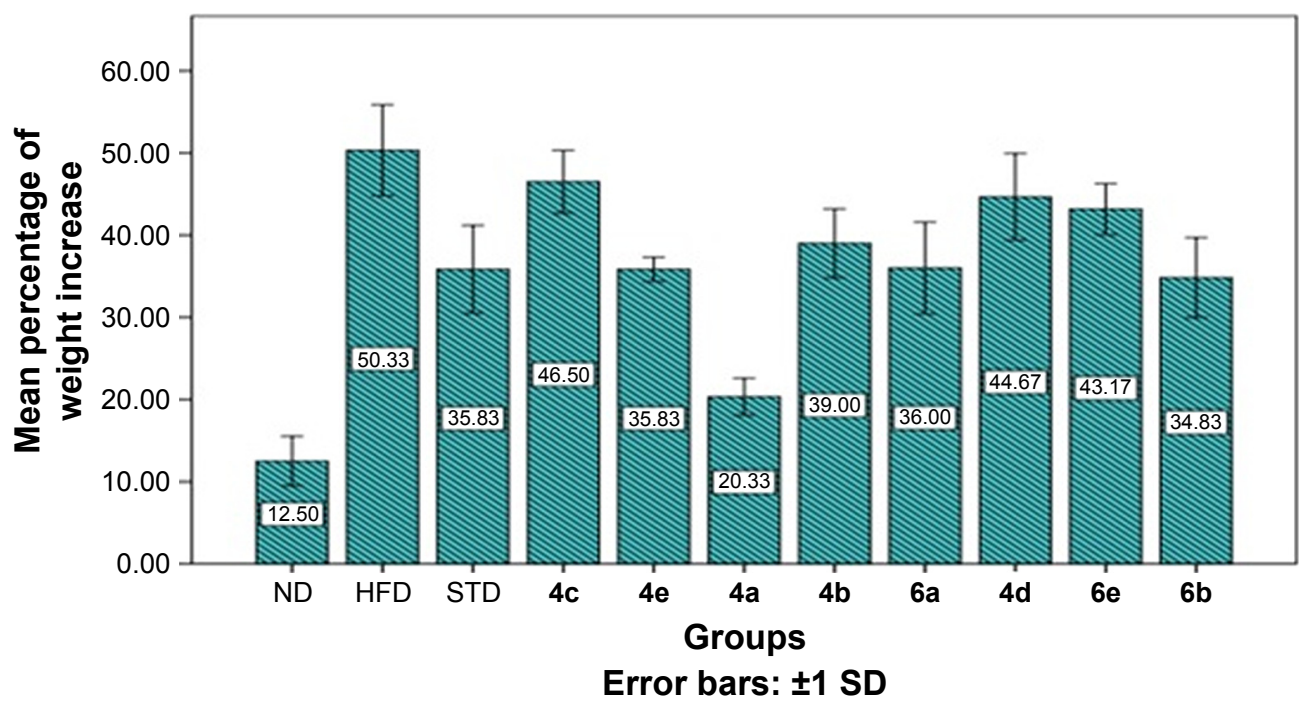

Figure 2 Effect of phenolic derivatives on body weight of mice fed on high-fat diet compared with standard (Atorvastatin) and high-fat diet alone. Note: Mean weight \pm SD ( $n=6$ in each group).

Abbreviations: ND, normal diet; HFD, high-fat diet; STD, reference standard (atorvastatin) 
Table 2 Effect of phenolic derivatives on lipid lowering efficiency in mice ( $n=6$, mean \pm SD) compared with normal control, hyperlipidemic control, and standard drug (atorvastatin)

\begin{tabular}{llllll}
\hline Group & $\begin{array}{l}\text { Dose } \\
(\mathbf{m g} / \mathbf{k g})\end{array}$ & $\begin{array}{l}\text { Cholesterol } \\
(\mathbf{m g} / \mathbf{d L})\end{array}$ & $\begin{array}{l}\text { TG } \\
(\mathbf{m g} / \mathbf{d L})\end{array}$ & $\begin{array}{l}\text { LDL } \\
(\mathbf{m g} / \mathbf{d L})\end{array}$ & $\begin{array}{l}\text { HDL } \\
(\mathbf{m g} / \mathbf{d L})\end{array}$ \\
\hline Normal control & - & $260 \pm 7.45$ & $127 \pm 5.10$ & $128 \pm 6.90$ & $82 \pm 4.14$ \\
Hyperlipidemic control & - & $386 \pm 12.93$ & $132 \pm 4.60$ & $317 \pm 7.09$ & $66 \pm 3.52$ \\
Atorvastatin & 10 & $165 \pm 7.54$ & $90 \pm 4.09$ & $89 \pm 4.73$ & $84 \pm 2.85$ \\
4a & 10 & $150 \pm 4.79 *$ & $84 \pm 4.09$ & $64 \pm 4.69^{* *}$ & $96 \pm 4.35^{*}$ \\
4b & 10 & $171 \pm 4.47$ & $86 \pm 5.10$ & $54 \pm 4.56^{* *}$ & $71 \pm 5.49$ \\
4c & 10 & $176 \pm 6.41$ & $86 \pm 4.43$ & $57 \pm 4.35$ & $69 \pm 4.64$ \\
4d & 10 & $179 \pm 7.49$ & $88 \pm 5.68$ & $61 \pm 3.89$ & $70 \pm 2.82$ \\
4e & 10 & $236 \pm 6.80$ & $95 \pm 4.16$ & $88 \pm 4.02$ & $67 \pm 4.16$ \\
6a & 10 & $148 \pm 4.38^{*}$ & $80 \pm 2.48 * *$ & $63 \pm 4.00$ & $68 \pm 8.37 * *$ \\
6b & 10 & $150 \pm 3.60 * *$ & $85 \pm 3.68$ & $56 \pm 4.85$ & $77 \pm 4.04^{* *}$ \\
6c & 10 & $156 \pm 3.65$ & $81 \pm 3.31 * *$ & $58 \pm 2.09$ & $80 \pm 3.46 * *$ \\
\hline
\end{tabular}

Notes: $* P<0.01$ vs standard drug (atorvastatin). $* * P<0.05$ vs standard drug (atorvastatin).

Abbreviations: TG, triglyceride; LDL, low-density lipoprotein; HDL, high-density lipoprotein.

cholesterol compared to standard with $P<0.01$, highly significant increase in HDL compared to standard with $P<0.01$, and significant reduction in LDL compared to standard with $P<0.05$ (Table 2). In order to unveil dose-dependent effect of $\mathbf{4 a}$, it was evaluated in same hyperlipidemic mice model with three different doses, ie, high dose $(10 \mathrm{mg} / \mathrm{kg})$, medium dose $(5 \mathrm{mg} / \mathrm{kg})$ and low dose $(2.5 \mathrm{mg} / \mathrm{kg})$. Effect of 4a reduced with decrease in dose when compared with high dose $10 \mathrm{mg} / \mathrm{kg}$, and this decrease was statistically significant (Table 3). In vivo outcomes clearly showing the superiority of antihyperlipidemic potential of $\mathbf{4 a}$ compared with other phenolic derivatives and even compared to standard atorvastatin. Furthermore, after 42 days of treatment none of the mice showed any kind of morbidity or mortality, which further advocates that all experimental phenolic compounds were well tolerated and were fairly safe during the whole experiment. These astounding outcomes establish the commendable and plausible antihyperlipidemic potential of our experimental natural phenolic derivatives.

\section{Histopathological examination}

Fatty liver is gaining importance in investigations and now is known to be most prevalent cause of abnormal liver.
Fatty liver initially starts with simple steatosis, which later may develop into advanced fibrosis and cryptogenic cirrhosis via steatohepatitis and ultimately to hepatocellular carcinoma. ${ }^{53}$ Obesity is the single main causative factor in the development of fatty liver both in children and adults. ${ }^{54}$ In our current investigation, $4 \mathbf{a}$ fed group of mice showed significant histopathological changes in liver sections under microscopic evaluation (Figure 3). A small loss of nucleus and membrane disruption was observed when compared with the normal diet (ND) group, but no fat droplet was observed in case of 4a group when compared with the HFD group. Histology of adipocytes showed that difference in size was evidently less in case of $\mathbf{4 a}$ group compared with the HFD group (Figure 3).

\section{Docking studies}

The in silico docking studies were performed to determine the binding affinity of the synthesized compounds with target protein. It has been reported that docking is an effective predictive tool regarding possible interactions of ligands with target protein. ${ }^{55}$ It further enables illustration of the possible atomic interactions between the particular functional group of a ligand in the active binding site of protein. To date, the

Table 3 Dose-dependent antihyperlipidemic effect of $\mathbf{4 a}(n=6$, mean \pm SD)

\begin{tabular}{|c|c|c|c|c|c|}
\hline Group & Dose (mg/kg) & $\begin{array}{l}\text { Cholesterol } \\
(\mathrm{mg} / \mathrm{dL})\end{array}$ & $\begin{array}{l}\text { TG } \\
(\mathrm{mg} / \mathrm{dL})\end{array}$ & $\begin{array}{l}\text { LDL } \\
(\mathrm{mg} / \mathrm{dL})\end{array}$ & $\begin{array}{l}\text { HDL } \\
(\mathrm{mg} / \mathrm{dL})\end{array}$ \\
\hline Normal control & - & $266 \pm 9.90$ & $12 \mid \pm 4.12$ & $124 \pm 3.79$ & $82 \pm 3.66$ \\
\hline Hyperlipidemic control & - & $380 \pm 10.84$ & $129 \pm 4.60$ & $314 \pm 7.09$ & $68 \pm 3.52$ \\
\hline $4 a$ & 2.5 (low dose) & $191 \pm 7.54^{*}$ & $94 \pm 4.09$ & $7 I \pm 4.73^{*}$ & $60 \pm 2.85$ \\
\hline $4 a$ & 5.0 (medium dose) & $|7| \pm 5.4 \mid * *$ & $90 \pm 4.13^{*}$ & $68 \pm 3.35$ & $76 \pm 3.64 *$ \\
\hline $4 a$ & I0 (high dose) & $|48 \pm 4.2|^{* *}$ & $87 \pm 3.89 *$ & $6 I \pm 3.6 I^{* *}$ & $96 \pm 4.12 * *$ \\
\hline
\end{tabular}

Notes: $* P<0.05$ vs hyperlipidemic group. $* * P<0.01$ vs hyperlipidemic group.

Abbreviations: TG, triglyceride; LDL, low-density lipoprotein; HDL, high-density lipoprotein. 


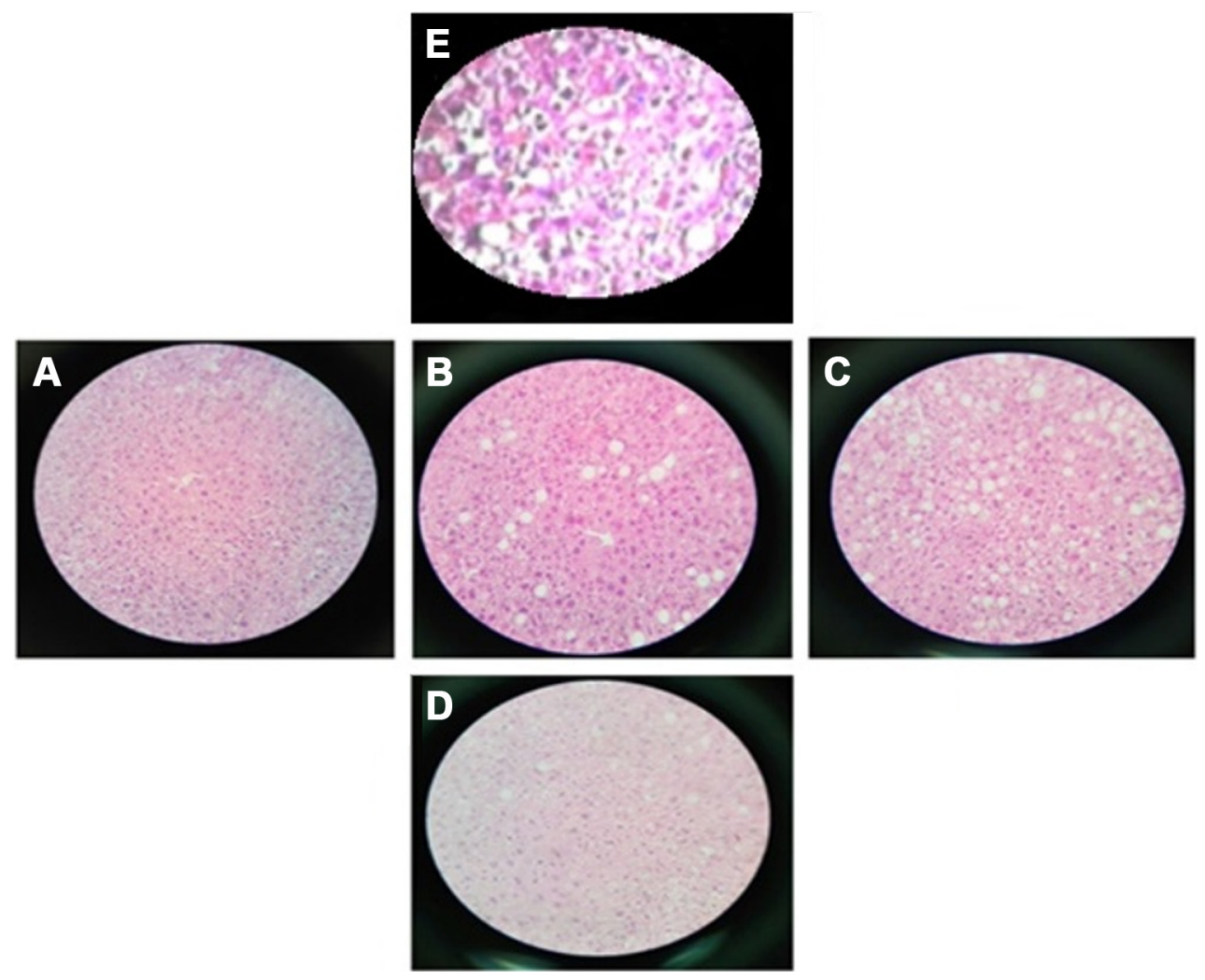

Figure 3 Histopathological evaluation of liver section slides: (A) 4a group; (B) 6a group; (C) 6b group; (D) standard drug group; and (E) hyperlipidemic control.

most successful strategy to control hyperlipidemic disorders has been to inhibit HMG CoA reductase. This enzyme catalyzes the conversion of HMG CoA to mevalonic acid, ultimately producing cholesterol. ${ }^{56}$ HMG CoA reductase model characterized with X-ray diffraction at a resolution of $2.22 \AA$ was downloaded from Protein Data Bank (Figure 4). The structure was validated by using the Ramachandran plot, which demonstrated that $>95.7 \%$ residues were lying in favorable region and $99.4 \%$ residues were lying in allowed region of plot (Figure 5). The experimental compounds (phenolic derivatives) tightly entrapped in active binding sites of HMG CoA reductase enzyme were visualized through Discovery Studio 4.1 (Figure 6). In the present study, we compared docking outcomes of our experimental phenolic compounds with atorvastatin (most widely used US Food and Drug Administration-approved statin), 4a and 6a showed even better binding affinity $(-8.3$ and $-7.9 \mathrm{kcal} /$ mol, respectively) energy values compared with atorvastatin $(-7.8 \mathrm{kcal} / \mathrm{mol})$ (Table 4). Analysis of residual interaction between inhibitor and target enzyme in active binding site revealed that the compound 4a interacted with Val805 (at a distance of $1.89 \AA$ ), Met656 (at a distance of $2.96 \AA$ ), Thr558 (at a distance of $2.70 \AA$ ), and Glu559 (at a distance of $2.20 \AA$ ) via hydrogen bonding in the active binding site of HMG CoA reductase (Figure 7). In contrast, 6a interacts with Asn658 via hydrogen bond at a distance of $3.06 \AA$ and with aspartate 767 at a distance of $4.33 \AA$ (Figure 8 ). These interaction patterns give a strong impression that synthesized phenolic derivatives possess good inhibitory potential against HMG CoA reductase.

\section{Conclusion}

The synthesis of some phenolic derivatives $4 \mathbf{a}-\mathbf{e}$ and $\mathbf{6 a}-\mathbf{c}$ was successfully accomplished starting with simplest precursors. The results of in silico docking studies showed that

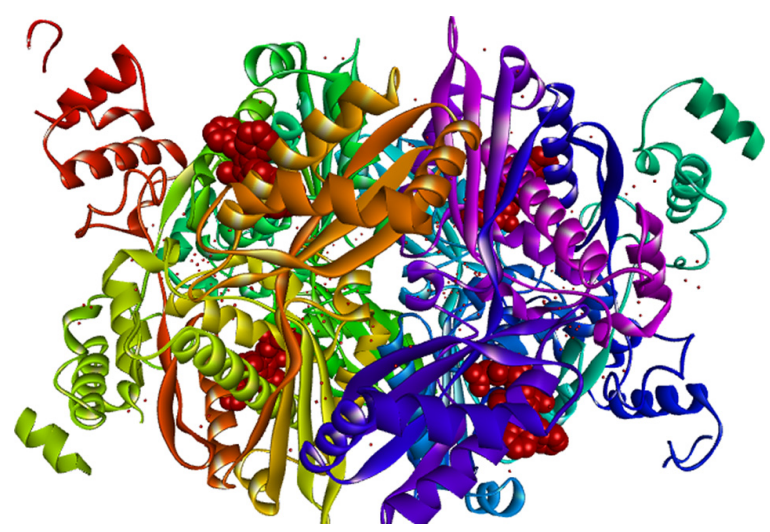

Figure 4 Three dimensional structure of homosapien HMG CoA reductase downloaded from Protein Data Bank with PDB ID IHWK bound to ligands. 


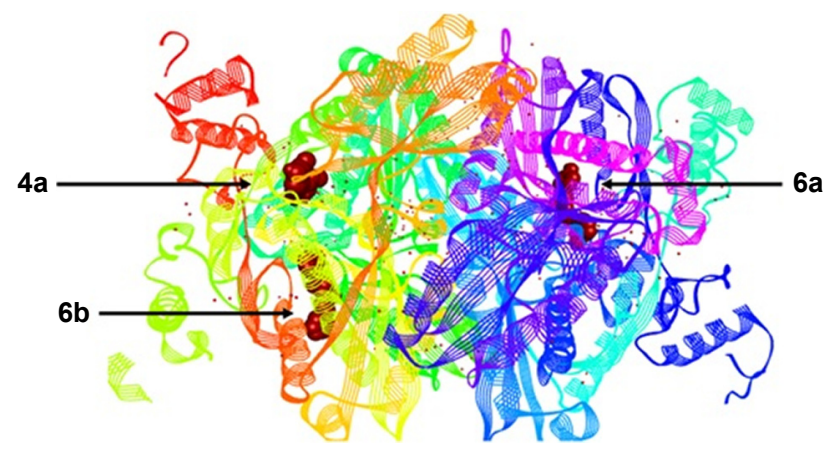

Figure 5 The phenolic derivatives $\mathbf{4 a}, \mathbf{6 a}$, and $\mathbf{6} \mathbf{b}$ tightly entrapped in active binding sites of HMG COA reductase enzyme visualized through Discovery Studio 4.I.

compounds $\mathbf{4 a}$ and $\mathbf{6 a}$ exhibited maximum binding affinity with target protein having binding energies -8.3 and $-7.9 \mathrm{kcal}$, respectively. Compound 4a interacts with amino acids Val805 having distance $1.89 \AA$ and Met656, Thr558, and Glu559 having bonding distances $2.96,2.70$, and $2.20 \AA$, respectively. Compound 4 a exhibited excellent in vivo antihyperlipidemic

\section{A}

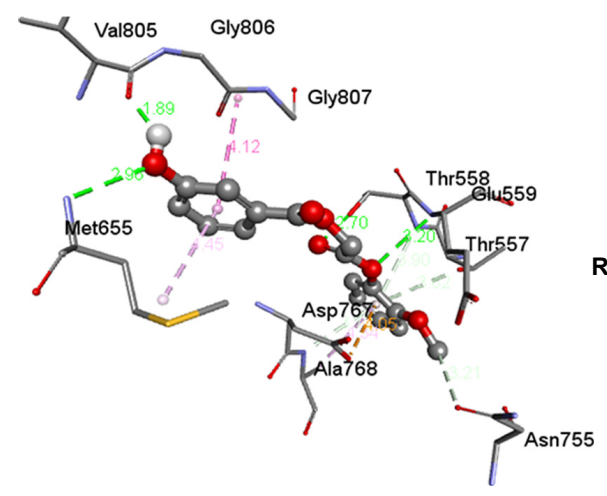

Residue interaction

Electrostatic

van der Waals

Covalent bond

Water

Metal

B

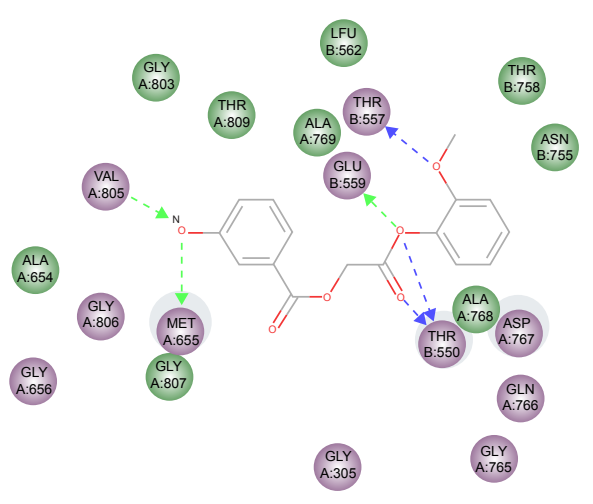

Figure 6 Residual interaction of 4a in binding pocket of HMG CO A reductase. Notes: (A) Shows three dimensional docking in active binding site. (B) Shows two dimensional interaction patterns. The legend inset represents the type of interaction between the ligand atoms and the amino acid residues of the protein. Compound $4 \mathrm{a}$ interacts via hydrogen bonding with $\mathrm{Val} 805$ (at a distance of $1.89 \mathrm{~A}^{\circ}$ ), Met 656 (at a distance of $2.96 \mathrm{~A}^{\circ}$ ), Thr 558 (at a distance of $2.70 \mathrm{~A}^{\circ}$ ), and Glu 559 (at a distance of $2.20 \mathrm{~A}^{\circ}$ ) amino acids.
Table 4 Binding affinity/energy values against HMG CoA reductase (PDB ID: IHWK)

\begin{tabular}{llll}
\hline Ligand & Binding affinity & RMSD/UB & RMSD/LB \\
\hline 4a & -8.3 & 0 & 0 \\
4b & -7.1 & 0 & 0 \\
4c & -7.1 & 0 & 0 \\
4d & -6.5 & 0 & 0 \\
4e & -6.4 & 0 & 0 \\
6a & -7.9 & 0 & 0 \\
6b & -7.4 & 0 & 0 \\
6c & -6.3 & 0 & 0 \\
Atorvastatin & -7.8 & 0 & 0
\end{tabular}

Note: Lesser binding affinity energy means less free energy of the interaction complex, ultimately illuminating that ligand-target complex is more stable.

Abbreviations: HMG CoA, 3-hydroxy-3-methylglutaryl-coenzyme A; RMSD, root mean square deviation; UB, upper bound; LB, lower bound.

activity with minimum weight increment (20\%) compared to standard atorvastatin (35\% weight increment). The synthesized compounds were nontoxic and well tolerated because none of the mice were found to suffer from any kind of

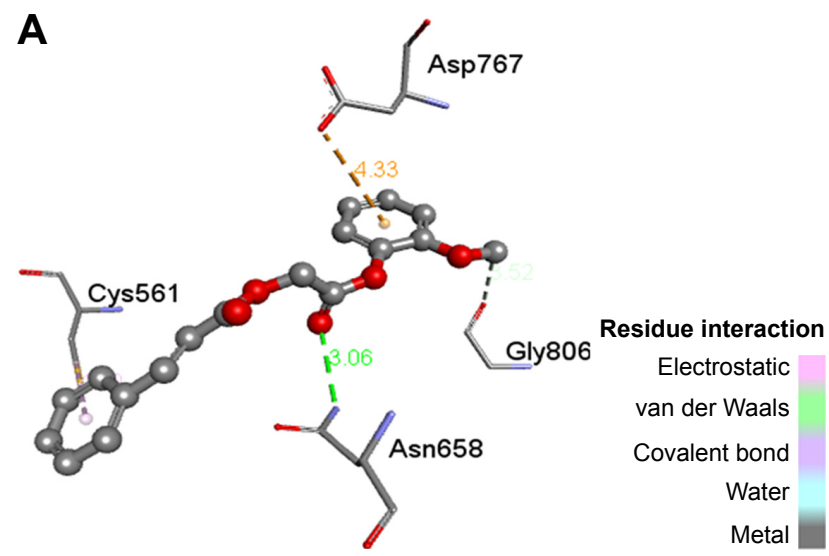

B

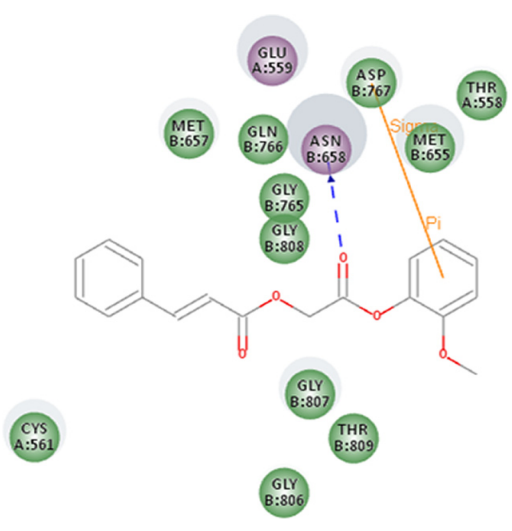

Figure 7 Residual interaction of $\mathbf{6 a}$ in binding pocket of HMG CO A reductase. Notes: (A) Shows three dimensional docking in active binding site. (B) Shows two dimensional interaction patterns. The legend inset represents the type of interaction between the ligand atoms and the amino acid residues of the protein. Compound $\mathbf{6 a}$ interacts via hydrogen bonding with ASN 658 (at a distance of $3.06 \mathrm{~A}^{\circ}$ ) and with ASP 767 (at a distance of $4.33 \mathrm{~A}^{\circ}$ ) amino acids. 


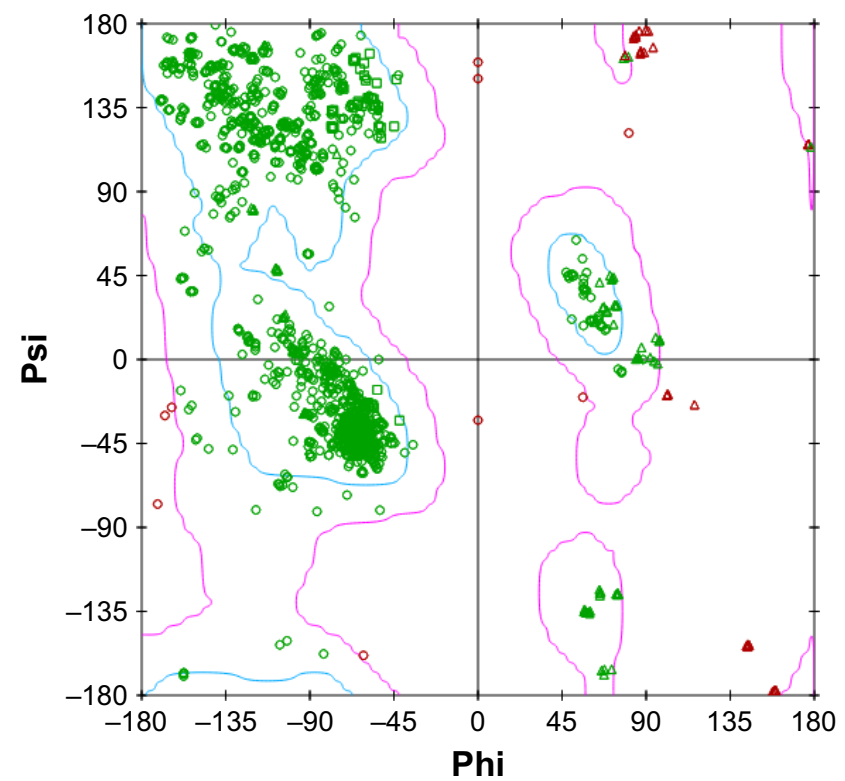

Figure 8 Ramachandran plot of protein structure of target protein (HMG COA reductase).

Notes: Total residues =1573; residues in favored region $=98 \%$; residues in allowed region $=99.4 \%$; total outliers $=9$.

morbidity and death during 6 weeks of dosing. Based on our pharmacological evaluation, we may propose that compound 4a may act as a lead structure for the design and development of more potent antihyperlipidemic drugs.

\section{Disclosure}

The authors report no conflicts of interest in this work.

\section{References}

1. Wani FA, Albahrawy AZ, Rahiman S. Hypolipidemic activity of olive oil (oleaeuropaea) against high fat diet-induced nonalcoholic fatty liver disease (NAFLD) in mice. Open J Path. 2015;5(3):73.

2. Meng XY, Zhang HX, Mezei M, Cui M. Molecular docking: a powerful approach for structure-based drug discovery. Curr Comput Aided Drug Des. 2011;7(2):146-157.

3. Kapetanovic IM. Computer-aided drug discovery and development (CADDD): in silico-chemico-biological approach. Chem Biol Interact. 2008;171(2):165-176.

4. Chiavari G, Vittorio C, Guido CG. Electrochemical detection in the high-performance liquid chromatographic analysis of plant phenolics. Analyst. 1988;113(1):91-94.

5. Rehman MR, Syed MAS, Maisam AS, et al. Comprehensive review on anti-oxidant activity of plants. Pak J Med Biol Sci. 2017;1(1):41-51.

6. Gan RY, Xiang RX, Feng LS, et al. Antioxidant activity and total phenolic content of medicinal plants associated with prevention and treatment of cardiovascular and cerebrovascular diseases. J Med Plant Res. 2010;4(22):2438-2444.

7. Rice E, Catherine A, Nicholas JM, et al. Structure-antioxidant activity relationships of flavonoids and phenolic acids. Free Radical Bio Med. 1996;20(7):933-956.

8. Skerget M, Kotnik P, Hadolin M, et al. Phenols, proanthocyanidins, flavones and flavonols in some plant materials and their antioxidant activities. Food Chem. 2005;89(2):191-198.
9. Tripoli E, Giammanco M, Tabacchi G, Di Majo D, Giammanco S, La Guardia M. The phenolic compounds of olive oil: structure, biological activity and beneficial effects on human health. Nutr Res Rev. 2005; 18(1):98-112.

10. Liu L, Zubik L, Collins FW, Marko M, Meydani M. The antiatherogenic potential of oat phenolic compounds. Atherosclerosis. 2004;175(1): 39-49.

11. Turner R, Nicolas E, Maria GA, et al. Antioxidant and anti-atherogenic activities of olive oil phenolics. Int J Vitam Nutr Res. 2005;75(1):61-70.

12. Negro C, Tommasi L, Miceli A. Phenolic compounds and antioxidant activity from red grape marc extracts. Bioresour Technol. 2003;87(1): 41-44.

13. Kelm MA, Nair MG, Strasburg GM, DeWitt DL. Antioxidant and cyclooxygenase inhibitory phenolic compounds from Ocimum sanctum Linn. Phytomedicine. 2000;7(1):7-13.

14. Zhang L, Ravipati AS, Koyyalamudi SR, et al. Antioxidant and anti-inflammatory activities of selected medicinal plants containing phenolic and flavonoid compounds. J Agric Food Chem. 2011;59(23): 12361-12367.

15. Conforti F, Sosa S, Marrelli M, et al. In vivo anti-inflammatory and in vitro antioxidant activities of Mediterranean dietary plants. J Ethnopharmacol. 2008;116(1):144-151.

16. Kassim M, Achoui M, Mustafa MR, Mohd MA, Yusoff KM. Ellagic acid, phenolic acids, and flavonoids in Malaysian honey extracts demonstrate in vitro anti-inflammatory activity. Nutr Res. 2010;30(9):650-659.

17. Han N, Gu Y, Ye C, Cao Y, Liu Z, Yin J. Antithrombotic activity of fractions and components obtained from raspberry leaves (Rubuschingii). Food Chem. 2012;132(1):181-185.

18. Kim MS, Kyung AL. Antithrombotic activity of methanolic extract of Umbilicaria esculenta. J Ethnopharmacol. 2006;105(3):342-345.

19. Carrieri C, Rosa Anna M, Francesca I, et al. Antithrombotic activity of 12 table grape varieties. Relationship with polyphenolic profile. Food Chem. 2013;140(4):647-653.

20. Hsu CL, Wu CH, Huang SL, Yen GC. Phenolic compounds rutin and o-coumaric acid ameliorate obesity induced by high-fat diet in rats. J Agric Food Chem. 2009;57(2):425-431.

21. Kaur G, Meena C. Evaluation of anti-hyperlipidemic potential of combinatorial extract of curcumin, piperine and quercetin in Triton induced hyperlipidemia in rats. Sci Int. 2013;1(3):57-63.

22. Chen CY, Milbury PE, Kwak HK, Collins FW, Samuel P, Blumberg JB. Avenanthramides and phenolic acids from oats are bioavailable and act synergistically with vitamin $\mathrm{C}$ to enhance hamster and human LDL resistance to oxidation. J Nutr. 2004;134(6):1459-1466.

23. Maurya DK, Devasagayam TP. Antioxidant and prooxidant nature of hydroxycinnamic acid derivatives ferulic and caffeic acids. Food Chem Toxicol. 2010;48(12):3369-3373.

24. Cheng JC, Fang D, Bo Z, et al. Antioxidant activity of hydroxycinnamic acid derivatives in human low-density lipoprotein: mechanism and structure-activity relationship. Food Chem. 2007;104(1):132-139.

25. Trombino S, Serini S, Di NF, et al. Antioxidant effect of ferulic acid in isolated membranes and intact cells: synergistic interactions with $\alpha$-tocopherol, $\beta$-carotene, and ascorbic acid. J Agric Food Chem. 2004; 52(8):2411-2420.

26. Rehman MR, Farhan S, Umbreen N, et al. Medicinal plants having wound healing activity; a review. Pak J Med Biol Sci. 2017;1(1):63-69.

27. Ou S, Kinchor K. Ferulic acid: pharmaceutical functions, preparation and applications in foods. J Sci Food Agric. 2004;84(11):1261-1269.

28. Cheng JC, Dai F, Zhou B, et al. Antioxidant activity of hydroxycinnamic acid derivatives in human low-density lipoprotein: mechanism and structure-activity relationship. Food Chem. 2007;104(1):132-139.

29. Chen JH, Chi-Tang H. Antioxidant activities of caffeic acid and its related hydroxycinnamic acid compounds. J Agric Food Chem. 1997;45(7): 2374-2378.

30. Gülçin İ. Antioxidant activity of caffeic acid (3, 4-dihydroxycinnamic acid). Toxicol. 2006;217(2):213-220. 
31. Lee YJ, Liao PH, Chen WK, Yang CY. Preferential cytotoxicity of caffeic acid phenethyl ester analogues on oral cancer cells. Cancer Lett. 2000; 153(1):51-56.

32. Fresco P, Borges F, Marques MP, Diniz C. The anticancer properties of dietary polyphenols and its relation with apoptosis. Curr Pharm Des. 2010;16(1):114-134

33. Huang MT, Smart RC, Wong CQ, Conney AH. Inhibitory effect of curcumin, chlorogenic acid, caffeic acid, and ferulic acid on tumor promotion in mouse skin by 12-O-tetradecanoylphorbol-13-acetate. Cancer Res. 1988;48(21):5941-5946.

34. Islam B, Sharma C, Adem A, Aburawi E, Ojha S. Insight into the mechanism of polyphenols on the activity of HMGR by molecular docking. Drug des Devel Ther. 2015;9:4943.

35. Ekins S, Jordi M, Bernard T. In silico pharmacology for drug discovery: methods for virtual ligand screening and profiling. Br J Pharmacol. 2007; 152(1):9-20.

36. Bender A, Josef S, Meir G, et al. Analysis of pharmacology data and the prediction of adverse drug reactions and off-target effects from chemical structure. ChemMedChem. 2007;2(6):861-873.

37. Bowes J, Brown AJ, Hamon J, et al. Reducing safety-related drug attrition: the use of in vitro pharmacological profiling. Nat Rev Drug Discov. 2012;11(12):909-922.

38. Kitchen DB, Decornez H, Furr JR, Bajorath J. Docking and scoring in virtual screening for drug discovery: methods and applications. Nat Rev Drug Discov. 2004;3(11):935-949.

39. Sashidhara KV, Kumar A, Kumar M, Srivastava A, Puri A. Synthesis and antihyperlipidemic activity of novel coumarin bisindole derivatives. Bioorg Med Chem Lett. 2010;20(22):6504-6507.

40. Jangad A, Periasamy S, Na YL, et al. Comparison of hypolipidemic activity of synthetic gallic acid-linoleic acid ester with mixture of gallic acid and linoleic acid, gallic acid, and linoleic acid on high-fat diet induced obesity in C57BL/6 Cr Slc mice. Chem Biol Interact. 2008;174(2): 109-117.

41. Rose PW, Beran B, Bi C, et al. The RCSB protein data bank: redesigned web site and web services. Nucleic Acids Res. 2010;39(1S):392-401.

42. Davis IW, Andrew LF, Vincent BC, et al. Mol Probity: all-atom contacts and structure validation for proteins and nucleic acids. Nucleic Acids Res. 2007;35(2S):W375-W383.

43. Goddard TD, Conrad CH, Thomas EF. Visualizing density maps with UCSF Chimera. J Struct Biol. 2007;157(1):281-287.
44. Morris GM, Huey R, Lindstrom W, et al. AutoDock4 and auto dock tools 4: automated docking with selective receptor flexibility. J Comput Chem. 2009;30(16):2785-2791.

45. Worachartcheewan A, Nantasenamat C, Naenna T, Isarankura-NaAyudhya C, Prachayasittikul V. Modeling the activity of furin inhibitors using artificial neural network. Eur J Med Chem. 2009;44(4): 1664-1673.

46. Forli S, Huey R, Pique ME, Sanner MF, Goodsell DS, Olson AJ. Computational protein-ligand docking and virtual drug screening with the auto dock suite. Nat Protoc. 2016;11(5):905-919.

47. Dallakyan S, Olson AJ. Small-molecule library screening by docking with PyRx. Methods Mol Biol. 2015;1263:243-250.

48. Ashraf Z, Alamgeer, Kanwal M, et al. Flurbiprofen-antioxidant mutual pro drugs as safer nonsteroidal anti-inflammatory drugs: synthesis, pharmacological investigation, and computational molecular modeling. Drug Des Dev Ther. 2016;10:2401-2419.

49. Bray GA, Fisler J, York DA. Neuroendocrine control of the development of obesity: understanding gained from studies of experimental animal models. Front Neuroendocrinol. 1990;11(2):128-181.

50. Langer T, Hoffmann RD. Virtual screening an effective tool for lead structure discovery. Curr Pharm Des. 2001;7(7):509-527.

51. Frostegard J. SLE, atherosclerosis and cardiovascular disease. J Intern Med. 2005;257(6):485-495.

52. Law MR, Wald NJ, Rudnicka AR. Quantifying effect of statins on low density lipoprotein cholesterol, ischaemic heart disease, and stroke: systematic review and meta-analysis. Br Med J. 2003;326(7404):1423.

53. Festi D, Colecchia A, Sacco T, Bondi M, Roda E, Marchesini G. Hepatic steatosis in obese patients: clinical aspects and prognostic significance. Obes Rev. 2004;5(1):27-42.

54. Wanless IR, Lentz JS. Fatty liver hepatitis (steatohepatitis) and obesity: an autopsy study with analysis of risk factors. Hepatology. 1990; 12(5):1106-1110

55. Istvan ES, Deisenhofer J. Structural mechanism for statin inhibition of HMG-CoA reductase. Science. 2001;292(5519):1160-1164.

56. Movahedian A, Sadeghi H, Ghannadi A, Gharavi M, Azarpajooh S. Hypolipidemic activity of Allium porrum L. in cholesterol-fed rabbits. J Med Food. 2006;9(1):98-101.

\section{Publish your work in this journal}

Drug Design, Development and Therapy is an international, peerreviewed open-access journal that spans the spectrum of drug design and development through to clinical applications. Clinical outcomes, patient safety, and programs for the development and effective, safe, and sustained use of medicines are the features of the journal, which

\section{Dovepress}

has also been accepted for indexing on PubMed Central. The manuscript management system is completely online and includes a very quick and fair peer-review system, which is all easy to use. Visit http://www.dovepress.com/testimonials.php to read real quotes from published authors. 